PAPER

\title{
Cognitive impairment and functional outcome after stroke associated with small vessel disease
}

\author{
V C T Mok, A Wong, W W M Lam, Y H Fan, W K Tang, T Kwok, A C F Hui, K S Wong
}

J Neurol Neurosurg Psychiatry 2004;75:560-566. doi: 10.1136/jnnp.2003.015107

See end of article for authors' affiliations

.....................

Correspondence to: Dr V C T Mok, Department of Medicine, Prince of Wales Hospital, Shatin, The Chinese University of Hong Kong, Hong Kong, China; vctmok@ netvigator.com

Received 25 March In revised form 20 July 2003

Accepted

7 September 2003

\begin{abstract}
Objectives: Although stroke associated with small vessel disease (SSVD) can induce both motor and cognitive impairment, the latter has received less attention. We aimed to evaluate the frequency of the varying severity levels of cognitive impairment, the determinants of severe cognitive impairment, and the association of cognitive impairment with functional outcome after SSVD.

Methods: Consecutive patients admitted to hospital because of SSVD were assessed at 3 months after stroke. We performed a semi-structured clinical interview to screen for cognitive symptoms. Severity of cognitive symptoms was graded according to the Clinical Dementia Rating Scale (CDR). Performance on psychometric tests (Mini-Mental State Examination, Alzheimer's Disease Assessment Scale (cognition subscale), Mattis Dementia Rating Scale (initiation/perseverence subscale; MDRS I/P)) of patients of different CDR gradings was compared with that of 42 healthy controls. Basic demographic data, vascular risk factors, stroke severity (National Institute of Health Stroke Scale; NIHSS), pre-stroke cognitive decline (Informant Questionnaire on Cognitive Decline in the Elderly; IQCODE), functional outcome (Barthel index; BI), Instrumental Activities Of Daily Living; IADL), and neuroimaging features (site of recent small infarcts, number of silent small infarcts, white matter changes) were also compared among the groups. Regression analyses were performed to find predictors of severe cognitive impairment and poor functional outcome. Results: Among the 75 included patients, 39 (52\%) complained of cognitive symptoms. The number of patients in each CDR grading was as follows: 39 (52\%) had a CDR of 0, 26 (34.7\%) had a CDR of 0.5, 10 (13.3\%) had a CDR of $\geqslant 1$. Pre-stroke IQCODE and previous stroke predicted CDR $\geqslant 1$. The NIHSS was associated with more impaired BI. The NIHSS and MDRS I/P contributed most to impaired IADL.

Conclusions: Half of the patients with SSVD complained of varying severity of cognitive problems 3 months after stroke. Pre-stroke cognitive decline and previous stroke predict severe cognitive impairment post stroke. Stroke severity and executive dysfunction contribute most to a poor functional outcome.
\end{abstract}

$\mathrm{S}$ mall, deep infarcts, also known as lacunar infarcts, and subcortical white matter changes (WMC) are manifestations of underlying cerebral small vessel disease. $^{12}$ Apart from sensorimotor disturbance, these ischaemic lesions commonly induce cognitive impairment due to disruption of the frontal-subcortical or medial temporal limbic circuits. ${ }^{3-5}$ The pattern of cognitive impairment commonly involves executive dysfunction, memory loss, or aphasia. ${ }^{167}$ The progression of the cognitive impairment can be insidious, stepwise, or both. ${ }^{8-10}$ Eventually, cognitive impairment in small vessel disease may progress from mild to severe cognitive impairment. ${ }^{8}$ Subcortical vascular dementia is the current terminology for severe cognitive impairment associated with small vessel disease. ${ }^{1}$ Given its strong vascular component, it is believed to be more preventable than dementia of the Alzheimer's type. ${ }^{11}$

Stroke is a common presentation of small vessel disease. Most studies on stroke associated with small vessel disease (SSVD) focus on motor impairment and mortality. ${ }^{12-15}$ Few studies have investigated the frequency of differering severity levels of cognitive impairment. Furthermore, the influence of cognitive impairment on the functional outcome after SSVD has rarely been studied. The objectives of this study were to evaluate: (a) the frequency and features of the varying severity levels of cognitive impairment, $(b)$ the determinants for severe cognitive impairment, and (c) the association between cognitive impairment and functional outcome after SSVD.

\section{METHODS}

We evaluated consecutive patients with or without previous stroke who were admitted to the acute stroke unit of the Prince of Wales Hospital because of stroke or transient ischaemic attack (TIA) from January to June $2002(\mathrm{n}=294)$. Brain computed tomography (CT) was performed on all patients within 24 hours of admission. Patients with intracerebral haemorrhage were excluded $(\mathrm{n}=37)$. T1 and T2 weighted magnetic resonance imaging (MRI) (1.5T scanner, Sonata; Siemens Medical System, Erlangen, Germany) and magnetic resonance angiography (MRA) were performed on 234 patients within 3 months of their stroke. A small infarct was defined as a well circumscribed lesion giving a hyperintense (T2 weighted) or hypointense (T1 weighted) signal on MRI, or a hypodense signal on CT, $0.2-2 \mathrm{~cm}$ size in all dimensions, located in the subcortical white and gray matter, cerebellar white matter, and brain stem. Small infarcts that could account for the present neurological deficits were considered to be relevant. WMC was defined on MRI as ill defined hyperintense areas $\geqslant 5 \mathrm{~mm}$ on T2 weighted

\footnotetext{
Abbreviations: $\mathrm{BI}$, Barthel Index; CDR, Clinical Dementia Rating Scale; $C T$, computed tomography; IADL, Instrumental Activities Of Daily Living; IQCODE, Informant Questionnaire on Cognitive Decline in the Elderly; MDRS I/P, Mattis Dementia Rating Scale (initiation/perseverence subscale); MRA, magnetic resonance angiography; MRI, magnetic resonance imaging; NIHSS, National Institute of Health Stroke Scale; SSVD, stroke associated with small vessel disease; TCD, transcranial Doppler; TIA, transient ischaemic attack; WMC, white matter changes
} 
imaging, and on CT as ill defined and moderately hypodense areas of $\geqslant 5 \mathrm{~mm}$. Transcranial Doppler (TCD) ultrasound (EME TC-2000) was performed to screen for intracranial large artery stenosis among patients with CT showing relevant small infarct but upon whom MRA could not be performed. Carotid duplex ultrasound (Philip SD800 ultrasound machine, $7.5 \mathrm{MHz}$ transducer) was also performed upon all patients with relevant small infarct to screen for extracranial carotid artery stenosis.

Patients with SSVD were potentially eligible for the study. We defined SSVD as patients having relevant small infarct with or without WMC. We excluded patients with relevant small infarcts that were associated with relevant intracranial large artery disease, extracranial carotid artery stenosis, cardiac embolic sources, and other miscellaneous causes. Intracranial large artery disease was considered to be relevant if the stenosis was at least moderate $(\geqslant 50 \%)$ in severity and if the infarct was located within its arterial supply. The methods of defining moderate stenosis via MRA and TCD have been described previously. ${ }^{16}{ }^{17}$ Extracranial carotid artery stenosis was considered to be relevant if the stenosis was $\geqslant 50 \%$ and the infarct was located within its arterial distribution. Patients were presumed to have cardiac embolic sources if they had concurrent presence or past history of atrial fibrillation $(\mathrm{AF})$, sick sinus syndrome, metallic heart valves, acute congestive failure, recent $(\leqslant 6$ weeks before stroke) myocardial infarction, atrial myxoma, or patent foramen ovale. Electrocardiography was performed on all patients. Transthoracic or transoesophageal echocardiography was not required to confirm the source of emboli. Patients were considered to have miscellaneous causes if they had the following diseases: inflammatory disorders (for example, systemic lupus erythematosus), carotid or vertebral artery dissection as suggested by history and vascular neuroimaging, recreational drug misuse (for example, cocaine), or haematological disorders (for example, thrombocytosis). We particularly screened for these conditions during the follow up at 3 months by the same neurologist (VCTM) via clinical history, laboratory investigations, and medical case notes.

Other exclusion criteria were: (a) cortical or large subcortical $(>2 \mathrm{~cm})$ infarcts; $(b)$ intracerebral haemorrhage; (c) clinical signs that could not be explained by the small infarct; (d) normal imaging; (e) non-ischaemic lesions, for example, tumour or demyelination; $(f)$ presence of relevant small infarcts but with unknown vascular aetiology because of absence of MRA, transcranial sonographic temporal window, or default imaging appointment; $(g)$ known preexisting dementing illnesses that were not due to Alzheimer's disease or vascular dementia, for example, chronic alcoholism; (h) major depression according to the Diagnostic and Statistical Manual of Mental Disorders, 4th edition; ${ }^{18}$ and (i) communication problems hindering participation in cognitive assessment, such as a language barrier, or severe visual or hearing loss. Patients with post-stroke aphasia were not excluded from our study. A qualified psychiatrist (W K Tang) excluded major depression 3 months after stroke.

Among all included patients, sites of the relevant small infarct were classified into cerebral white matter (corona radiata and centrum semi ovale), striatocapsule, thalamus, cerebellum, and brainstem, based on neuroimaging findings with reference to the clinical presentation. The total number of silent small infarcts and severity of WMC were also recorded. Severity of WMC was graded according to the method reported by Wahlund et al. ${ }^{19}$ Briefly, WMC was graded in five different brain regions, frontal, parietooccipital, temporal, basal ganglia, and infratentorial, based on T2 weighted MRI or CT. The score for each region varied from 0 (no lesions) to 3 (diffuse involvement).
The total score was the sum of the grade given for each region. The same neurologist (YHF) graded the WMC, and another radiologist (WWML) provided other details of the neuroimaging while blinded to the patients' cognitive status.

\section{Cognitive assessment}

The same neurologist (VCTM) performed a semi-structured clinical interview on all patients to assess post-stroke cognition at 3 months after the stroke. During the semistructured clinical interview, both the patients and close informants were asked separately whether the patients noted any cognitive symptoms after the index stroke. Following this open ended question, specific questions were asked to screen for the presence of the following cognitive symptoms: memory loss, slow thinking (delay in decision making or answering questions), disorientation (in time, place, or person), aphasia (word finding difficulties, lack of fluency in speech, or difficulties in comprehension), and disinhibition (impulsive or inappropriate behaviour). Any types of cognitive symptoms that still remained in the week before the interview were considered to be relevant. Cognitive symptoms that increased following the stroke were also noted. The interference of the cognitive symptoms on patients' various aspects of daily function was then graded according to the $\mathrm{CDR}^{20}{ }^{21}$ during the same interview. The CDR evaluated patient's performance in six categories of cognitive functioning: memory, orientation, judgement and problem solving, community affairs, home and hobbies, and personal care. The CDR scoring system was based on Morris. ${ }^{21}$ There are five grades in the CDR: $0,0.5,1,2$, and 3; the higher the grade, the greater the cognitive impairment. In grading impairment in each of the categories, care was taken to grade only those impairments that were attributed to cognitive symptoms, not to motor or mood disturbances. Apart from the semi-structured clinical interview, the following psychometric tests were also administered to all patients: Chinese versions of the Mini-Mental State Examination (MMSE), ${ }^{22}$ Alzheimer's Disease Assessment Scale (cognition; ADAS$\operatorname{cog}),{ }^{23}$ and the Mattis Dementia Rating Scale (initiation/ perseverance subscale; MDRS I/P). ${ }^{24}$ The MMSE served as a brief global cognitive measure. The ADAS-cog is a more extensive measure than MMSE. It evaluates memory, orientation, language, construction, and ideational apraxia. The MDRS I/P has been used in other studies as a brief evaluation of executive dysfunction. ${ }^{525}$ The same research assistant (AW) administered the psychometric tests on all patients while blinded to the patients' CDR grading. The control group for the performance of psychometric tests comprised 42 healthy elderly people who were recruited from the following sources: community elderly centre $(n=31)$, relatives of hospital staff $(n=6)$, and spouses of patients $(n=3)$, and hospital staff $(n=2)$. The criteria for controls were absence of neurological or psychiatric diseases, or any cognitive symptoms, independence in daily functioning, and a CDR of 0. Again, the Chinese versions of the MMSE, ADAS$\operatorname{cog}$, MDRS I/P, BI, and IADL were administered. All controls and patients gave their informed consent to participate in this study.

Pre-stroke cognitive decline was assessed via the Chinese version of the Informant Questionnaire on Cognitive Decline in the Elderly (IQCODE) ${ }^{26}$ to the patients' closest available relatives within 2 weeks after the onset of the index stroke. The IQCODE contains 26 items that rate changes in memory and other cognitive abilities that have occurred in the previous 10 years. The informants were asked by a same research assistant to rate the changes on a 5 point scale ranging from "much better" to "much worse". 


\section{Clinical profile and functional outcome}

Data on age, gender, years of education, and vascular risk factors including hypertension (HT), diabetes mellitus (DM), heart diseases (coronary artery disease, heart failure, or atrial fibrillation), previous stroke or TIA, smoking, alcohol intake, and fasting lipids level were collected during the acute admission. Hypercholesterolaemia was defined as total cholesterol of $>6.2 \mathrm{mmol} / \mathrm{L}$ or low-density lipoprotein of $>4.1 \mathrm{mmol} / \mathrm{L}$. We performed the National Institute of Health Stroke Scale (NIHSS) during the first few days after stroke to measure patients' worst stroke severity. It mainly evaluates motor impairment (weakness, ataxia), sensory loss, visual field defects, and cortical deficits (aphasia, neglect). We determined the functional outcome of the patients at 3 months after stroke using the Barthel (BI) Index and Lawton IADL. ${ }^{27}$ Two research nurses who were blinded to patients' CDR grading and NIHSS score administered the scales. The BI and IADL evaluate basic and complex ADL respectively. The total score for BI is 20 , with a higher score representing better function. The score for each item of the IADL ranges from 0 to 3, with a lower score representing better function. As some items in the IADL were not scored because these items were not applicable to all patients owing to personal habits or motor impairment, we took the average of all scored items as the final IADL score.

\section{Statistical analysis}

Student's $t$ test and $\chi^{2}$ test were used for comparing continuous and dichotomous variables respectively between patients and controls. Analysis of covariance, using age and education as covariates, was used to compare psychometric scores of controls and patients. To assess the frequency of the varying severity of cognitive impairment, we divided the patients into three groups based on CDR: $0,0.5$, and $\geqslant 1$. We grouped those with CDRs of 1, 2, and 3 into one group for statistical analysis as there were only few patients who had $\mathrm{CDR} \geqslant 1$. Analysis of variance was used to compare all continuous variables of controls and the three cognitive groups. Post hoc tests with Bonferroni adjustment were performed with each analysis of variance. Because the neuroimaging scores were not normally distributed, MannWhitney U tests with Bonferroni correction were used for comparisons between the three cognitive groups. Variables that were significantly different $(\mathrm{p}<0.05)$ between patients with $\mathrm{CDR} \geqslant 1$ and $\leqslant 0.5$ in the binomial univariate analysis were entered into a forward stepwise multivariate binomial regression analysis to find independent predictors of CDR $\geqslant 1$. We also performed two univariate linear regression analyses to find the variables that accounted for the variance in BI and IADL respectively. Variables that were identified as significant in the univariate analysis $(\mathrm{p}<0.05)$ were entered into the forward stepwise multivariate linear regression analyses to examine their independent contributions to the variance of both BI and IADL.

\section{RESULTS}

Among the 294 admitted patients, 106 had relevant small infarcts. Among these 106 patients, 20 were excluded because of underlying relevant intracranial large artery disease $(n=14)$ and unknown vascular aetiology $(n=6)$. Two of the 14 patients with intracranial large artery disease also had concurrent relevant extracranial carotid artery disease. No relevant small infarcts were related to cardioembolism, lone relevant extracranial carotid disease, or other miscellaneous causes in our present cohort. Among the 86 patients with SSVD, 11 more patients were excluded from further cognitive assessment because of severe depression $(\mathrm{n}=3)$, death $(\mathrm{n}=1)$, default follow up $(\mathrm{n}=4)$, chronic alcoholism $(n=1)$, and language barrier $(n=2)$. Seventy five patients with SSVD (25.5\%) underwent cognitive assessment and were included in the final analysis. Comparison of the 75 included patients with the 17 excluded patients with relevant small infarcts but who had unknown vascular aetiology $(\mathrm{n}=6)$ or who did not undergo cognitive assessment $(\mathrm{n}=11)$ showed that the excluded patients were older ( 77 years) and had more severe stroke $($ NIHSS $=9.1$ ) than those who were included in the study (70.7 years old, $\mathrm{p}=0.006$; NIHSS $=4.3, \mathrm{p}=0.025$ ). Comparisons of controls and included patients showed no difference in terms of basic demographic data. The performance on MMSE, ADAS-cog, and MDRS I/P of the patients was significantly worse than that of controls after adjustment for age and education (table 1).

Among the 75 patients, 39 (52\%) complained of cognitive symptoms post-stroke. These cognitive symptoms represented either new onset or further increase in pre-stroke cognitive symptoms among $32(82.1 \%)$ of these 39 patients. The majority of the patients $(n=36 ; 92.3 \%)$ complained of memory problems. Slow thinking, disorientation, and aphasia occurred in $29(74.4 \%), 7(17.9 \%)$, and 7 (17.9\%) patients respectively. Disinhibition was noted only in one patient $(2.6 \%)$. No patients complained of other cognitive symptoms, such as neglect or apraxia, in response to the open ended question. Twenty nine patients $(74.4 \%)$ had more than one type of cognitive symptoms. Seven patients (17.9\%) had isolated memory complaints. Three patients $(7.7 \%)$ complained of slow thinking without associated memory complaints or other cognitive symptoms. No patients had isolated aphasia or disorientation. Among patients with SSVD, the number of patients in each CDR grading was as follows: 39 (52\%) had CDR of 0, 26 (34.7\%) had CDR of 0.5, 8 ( $10.7 \%)$ had CDR of 1,1 ( $1.3 \%)$ had CDR of 2 , and 1 (1.3\%) had CDR of 3. The three patients who complained of isolated slow thinking were classified as CDR of 0 according to the rating algorithm. ${ }^{21}$

Comparison of patients with varying CDR grading revealed that patients with $C D R \geqslant 1$ had significantly more impaired IADL, pre-stroke IQCODE, and performance on psychometric tests than those with $\mathrm{CDR}<1$, even though the groups had similar basic demographic data, clinical features, sites of recent infarcts, number of silent infarcts, and BI (table 2). Although patients with $C D R \geqslant 1$ also had more WMC and previous stroke than patients with $\mathrm{CDR}<\mathrm{l}$, these differences did not reach statistical significance. Patients with CDR of 0.5 had significantly more impaired pre-stroke IQCODE than those with CDR of 0 . Patients with CDR of 0 performed significantly worse on MDRS I/P and ADAS-cog than did controls even though they had similar basic demographic features.

Univariate regression analysis showed that pre-stroke IQCODE, NIHSS, previous stroke, and WMC were associated

\begin{tabular}{|c|c|c|c|}
\hline & $\begin{array}{l}\text { Controls } \\
(n=42)\end{array}$ & $\begin{array}{l}\text { Patients } \\
(n=75)\end{array}$ & $\mathbf{p}$ \\
\hline Gender (\% F) & 58.5 & 48 & 0.549 \\
\hline Age & $69.6(9.9)$ & 71.0 (11.2) & 0.095 \\
\hline Education & $5.4(4.6)$ & $4.8(4.1)$ & 0.086 \\
\hline $\mathrm{Bl}$ & $20(0)$ & $18.3(3.5)$ & $<0.001$ \\
\hline IADL & $0.1(0.2)$ & $0.9(0.8)$ & $<0.001$ \\
\hline MMSE & $27.7(2.1)$ & $24.8(4.5)$ & $<0.001$ \\
\hline MDRS I/P & $34.1(3.0)$ & $28.5(6.4)$ & $<0.001$ \\
\hline ADAS-cog & $9.5(3.4)$ & 16.5 (9.3) & $<0.001$ \\
\hline
\end{tabular}




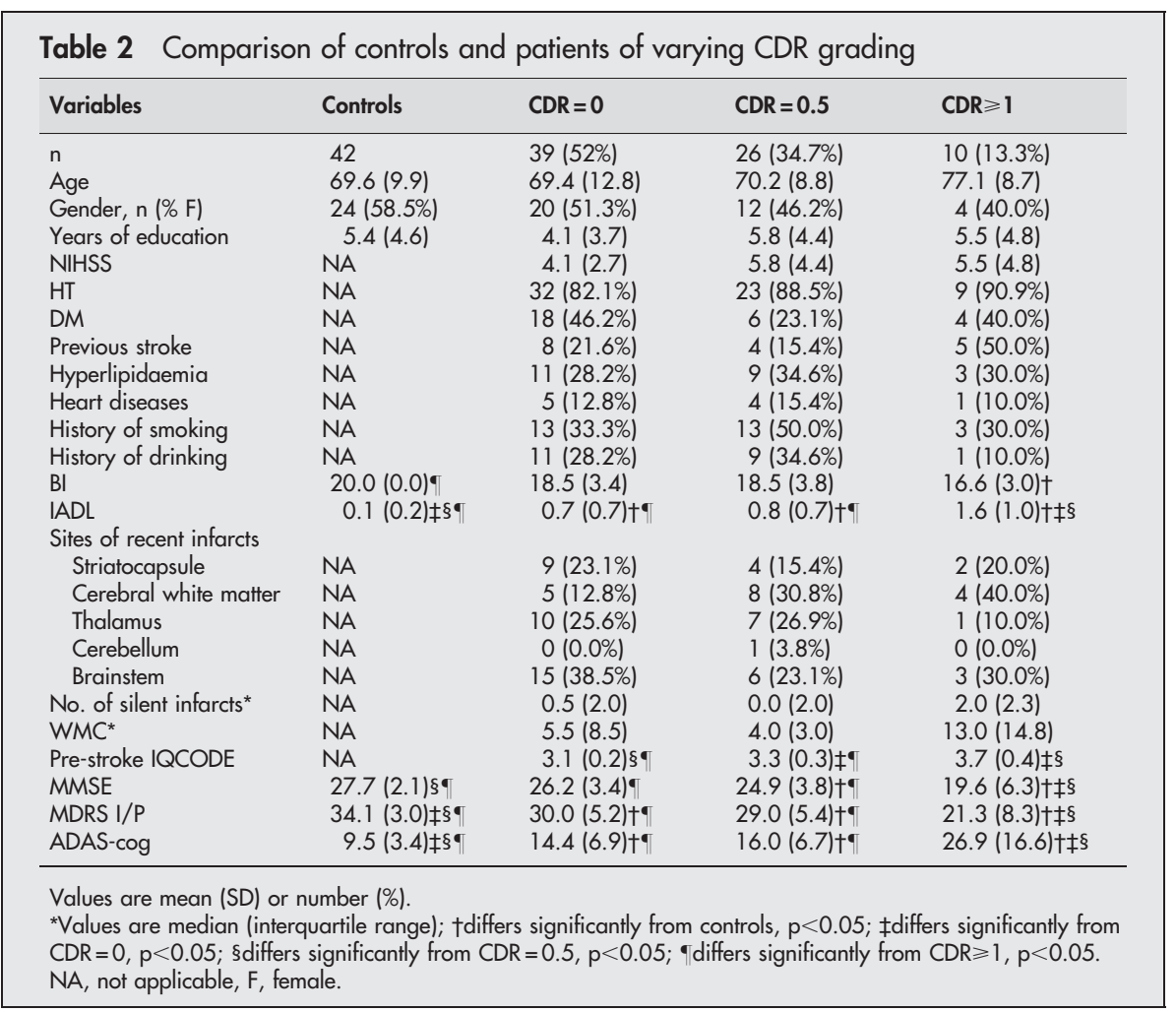

with $C D R \geqslant 1$ (table 3 ). Multivariate regression analysis only showed that pre-stroke IQCODE (odds ratio $(\mathrm{OR})=95.52$, $95 \%$ confidence interval $(\mathrm{CI})=7.37$ to $1248.42, \mathrm{p}<0.001)$ and previous stroke $(\mathrm{OR}=11.35,95 \% \mathrm{CI}=1.62$ to 79.73 , $\mathrm{p}=0.015$ ) significantly predicted $\mathrm{CDR} \geqslant 1$ (table 3 ).

Univariate regression analysis revealed that NIHSS, performance on MDRS I/P, and ADAS-cog were associated with BI. Multivariate regression analysis revealed that only NIHSS $\left(\mathrm{R}^{2}=0.096, \mathrm{p}=0.011\right)$ contributed significantly to the variance of BI (table 4). Univariate analysis revealed that age, education, NIHSS, pre-stroke IQCODE, WMC, and performance on all the three psychometric tests were associated with IADL. Multivariate regression analysis revealed that NIHSS $\left(\mathrm{R}^{2}=0.327, \mathrm{p}<0.001\right)$, performance on MDRS I/P $\left(\mathrm{R}^{2}=0.139, \mathrm{p}<0.001\right)$, age $\left(R^{2}=0.052, p=0.011\right)$, and pre-stroke IQCODE $\left(R^{2}=0.034\right.$, $\mathrm{p}=0.034)$ contributed significantly to the variance of IADL (table 4).

\section{DISCUSSION}

In this hospital based study, half of the patients with SSVD had varying severity of cognitive symptoms 3 months after SSVD. Pre-stroke cognitive decline and previous stroke predicted severe cognitive impairment. Stroke severity was associated with impaired basic ADL. Stroke severity and performance on MDRS I/P contributed most to an impaired complex ADL 3 months after SSVD.

Although small vessel disease is the most common cause for small infarct, other vascular aetiologies are also recognised as causing small infarct. ${ }^{28}{ }^{29}$ In order to include patients with SSVD, we had rigorously screened out other relevant vascular aetiologies. In particular, we had looked for relevant intracranial large artery disease, which is not uncommonly associated with small infarct in our ethnic group. ${ }^{16}$ Indeed, 14 patients $(13.2 \%)$ with relevant small infarct were found to have relevant intracranial large artery disease and were thus excluded. As patients who had relevant small infarcts with unknown vascular aetiology were also excluded, the subjects in our study probably represent patients with a homogeneous aetiology, namely small vessel disease.

In the present study, we attempted to describe cognitive impairment among patients with SSVD from three different perspectives: (a) cognitive symptoms as noted by the patients or their close informants, $(b)$ varying severity of the cognitive symptoms as graded by the CDR, and $(c)$ performance in various psychometric tests.

\section{Cognitive symptoms}

Cognitive symptoms were noted by more than half (52\%) of the patients 3 months after SSVD. Majority of the patients $(82.1 \%)$ claimed that there was a clear temporal relation between the cognitive symptoms and the index stroke. This is consistent with most studies showing that small infarcts

\begin{tabular}{|c|c|c|c|c|}
\hline \multirow[b]{2}{*}{ Statistical analysis } & \multirow[b]{2}{*}{$\mathbf{p}$} & \multirow[b]{2}{*}{$\mathrm{OR}^{*}$} & \multicolumn{2}{|l|}{$95 \% \mathrm{Cl}$} \\
\hline & & & Lower & Upper \\
\hline \multicolumn{5}{|l|}{ Univariate binomial regression } \\
\hline Age & NS & 1.08 & 1.00 & 1.17 \\
\hline Gender & NS & 0.69 & 0.18 & 2.67 \\
\hline Years of education & NS & 1.04 & 0.89 & 1.22 \\
\hline Pre-stroke IQCODE & $<0.01$ & 41.59 & 4.82 & 358.57 \\
\hline NIHSS & 0.05 & 1.29 & 1.00 & 1.66 \\
\hline HT & NS & 1.64 & 0.19 & 14.38 \\
\hline DM & NS & 1.14 & 0.29 & 4.45 \\
\hline Previous stroke & 0.04 & 4.25 & 1.06 & 17.06 \\
\hline Hyperlipidemia & NS & 0.96 & 0.23 & 4.12 \\
\hline Heart diseases & NS & 0.69 & 0.08 & 6.13 \\
\hline History of smoking & NS & 0.64 & 0.15 & 2.72 \\
\hline History of alcohol drinking & NS & 0.25 & 0.03 & 2.11 \\
\hline Total no. silent infarcts & NS & 1.30 & 0.82 & 2.05 \\
\hline WMC & 0.01 & 1.26 & 1.07 & 1.48 \\
\hline \multicolumn{5}{|c|}{ Stepwise multivariate binomial regression } \\
\hline Pre stroke IQCODE & $<0.001$ & 95.52 & 7.37 & 1238.42 \\
\hline Previous stroke & 0.015 & 11.35 & 1.62 & 79.73 \\
\hline
\end{tabular}


Table 4 Association of clinical, cognitive, and radiological features with functional outcome

\begin{tabular}{|c|c|c|c|c|}
\hline \multirow[b]{2}{*}{ Statistical analysis } & \multicolumn{2}{|l|}{ BI } & \multicolumn{2}{|l|}{ IADL } \\
\hline & $\mathrm{R}^{2}$ & $p$ (F change) & $\overline{R^{2}}$ & $p$ (F change) \\
\hline \multicolumn{5}{|c|}{ Univariate linear regression } \\
\hline Age & 0.003 & NS & 0.265 & $<0.001$ \\
\hline Education & 0.004 & NS & 0.142 & $<0.001$ \\
\hline NIHSS & 0.084 & 0.015 & 0.278 & $<0.001$ \\
\hline Pre-stroke IQCODE & 0.04 & NS & 0.236 & $<0.001$ \\
\hline MMSE & 0.036 & NS & 0.278 & $<0.001$ \\
\hline MDRS I/P & 0.098 & 0.009 & 0.285 & $<0.001$ \\
\hline ADAS-cog & 0.072 & 0.026 & 0.244 & $<0.001$ \\
\hline Previous stroke & 0.013 & NS & 0.001 & NS \\
\hline Heart disease & 0.005 & NS & $<0.001$ & NS \\
\hline No. of silent infarcts & 0.005 & NS & 0.035 & \\
\hline WMC & 0.007 & NS & 0.177 & $<0.001$ \\
\hline \multicolumn{5}{|c|}{ Stepwise multivariate linear regression } \\
\hline NIHSS & 0.096 & 0.011 & 0.327 & $<0.001$ \\
\hline MDRS I/P & NA & NA & 0.139 & $<0.001$ \\
\hline Age & NA & NA & 0.052 & 0.011 \\
\hline Pre-stroke IQCODE & NA & NA & 0.034 & 0.034 \\
\hline $\begin{array}{l}\text { Total variance } \\
\text { explained }\end{array}$ & $9.60 \%$ & & $55.20 \%$ & \\
\hline
\end{tabular}

located in various subcortical or infratentorial regions are capable of causing cognitive impairment as well as sensorimotor deficits..$^{3} 7^{30-33}$ As many of these patients already had varying degrees of pre-stroke cognitive decline, as suggested by their impaired pre-stroke IQCODE (table 2), the recent small infarct probably induced only a further stepwise deterioration of pre-existing cognitive impairment, rather than inducing a new onset cognitive impairment. The magnitude of cognitive deterioration that was associated with the recent small infarct was not ascertained in this study. Small case series suggest that the magnitude of cognitive deterioration may vary from subtle ${ }^{30}$ to severe. ${ }^{3}$

Our attempt to classify the types of cognitive impairment based on subjective symptoms was only exploratory. Patient complaint of memory loss may in fact be due to slow thinking or word finding difficulty; the reverse may also be true. Therefore, further study is needed to investigate the type of cognitive impairment by psychometric tests that evaluate specific cognitive domains.

\section{CDR grading and performance of psychometric tests}

To assess the frequency of the varying severity of cognitive impairment, we arbitrarily divided our patients into three 3 groups based on the CDR grading. To date, there is no standardised method to define the varying severity of cognitive impairment associated with cerebrovascular disease. ${ }^{34}$ We did not use cutoff scores of psychometric tests to grade cognitive impairment because controversy still exists in the selection of such tests. Furthermore, valid cutoff scores for defining different severity of cognitive impairment have not been determined in our ethnic group for this disease entity. We had chosen CDR simply because of its ease of administration and because other recent studies had also applied CDR in grading cognitive impairment associated with small vessel disease. ${ }^{25}{ }^{35}$ As CDR was originally designed for grading dementia of the Alzheimer's type, ${ }^{20}{ }^{21}$ memory bears greater weight in the rating algorithm. Thus, it may not reflect the true impact of non-memory cognitive symptoms that are associated with SSVD. In fact, the three patients with isolated slow thinking were graded as CDR of 0 according to the rating algorithm. Therefore, caution should be taken in interpreting the grading of the patients. However, given this limitation, we found that the performance in various psychometric tests of our patients correlated roughly with the severity of CDR grading (table 2). This correlation lends support to the validity of this functional based rating scale in estimating the severity of cognitive impairment in patients with SSVD. Minor modification by including symptoms of executive dysfunction such as slow thinking as a primary category may improve the sensitivity and accuracy of CDR in grading cognitive impairment in SSVD.

The frequency of $C D R \geqslant 1$ or those with greater cognitive impairment was $13.3 \%$. A few hospital based studies demonstrate that the frequency of dementia after SSVD ranges from $5 \%$ to $27 \%$ at 1 month to 3 years after SSVD $36-40$ (table 5). Our frequency of $13.3 \%$ fell within this range. The differences between the studies are probably caused by differences in the study population, method of defining dementia or severe cognitive impairment, and follow up time (table 5). The frequency of severe cognitive impairment in SSVD is less than that in large arterial stroke $(20 \%-32 \%) .3640$ We must emphasise that patients with severe cognitive impairment had only mild stroke severity (mean NIHSS of 5.5). However, they had significantly more impaired functioning in complex ADL than those with lesser cognitive impairment even though stroke severity and functioning in basic ADL were similar. These observations suggest that ignoring the cognitive impact among patients with severe cognitive impairment may underestimate the consequence of SSVD if motor deficits and basic ADL only are assessed in this group of patients.

Patients with CDR of 0.5 were almost identical to patients with CDR of 0 in terms of basic demographic data, stroke severity, vascular risk factors, neuroimaging features, poststroke functional impairment, and performance in all psychometric tests. Interestingly, we found that only prestroke cognitive decline was significantly more impaired in patients with CDR of 0.5 than that in patients with CDR of 0 even though both groups had similar performance in psychometric tests. There are several possibilities that may explain the subtle difference between these two groups. Firstly, as addressed above, the CDR grading may undergrade the cognitive consequence of patients with mild, nonmemory cognitive symptoms. Including these patients into CDR of 0 may mask any genuine differences between the two groups. Secondly, the various psychometric tests that were used in our study may not be sensitive enough to reveal a significant difference between these two groups of patients. Thirdly, the pre-stroke IQCODE may be more sensitive in detecting mild post-stroke cognitive impairment in patients with SSVD than other psychometric tests that were used in our study. Fourthly, the sample size may not be powerful enough to demonstrate a statistical difference in the performance of psychometric tests between the two groups. The mean scores of all psychometric tests for patients with CDR of 0.5 appeared to be slightly worse than that for patients with CDR of 0 . A larger sample size may possibly reveal a statistical difference in the performance of psychometric tests between these two groups of patients. Lastly, any combinations of the above may be responsible.

Apart from the three patients who had mild isolated slow thinking, all the remaining patients with CDR of 0 denied any cognitive symptoms before or after the stroke. Their performances in MDRS I/P and ADAS-cog were significantly worse than that of controls even though both groups had similar basic demographic data. We postulate that this difference is probably due to the small infarcts and WMC. Studies demonstrate that both small subcortical infarcts and WMC are associated with impaired performance in psychometric tests among healthy individuals who are free of stroke or cognitive symptoms. ${ }^{51}$ We did not perform neuroimaging for our controls. Silent infarcts and age related WMC might 


\begin{tabular}{|c|c|c|c|c|c|}
\hline Studies & $\mathbf{n}$ & Study population & Dementia criteria & $\begin{array}{l}\text { Follow up } \\
\text { duration }\end{array}$ & $\begin{array}{l}\text { Frequency } \\
(\%)\end{array}$ \\
\hline Tatemichi $^{36}$ & 227 & $\begin{array}{l}\text { With/without previous stroke } \\
\text { or pre-stroke dementia }\end{array}$ & Clinical diagnosis & 2 years & 11 \\
\hline Wolfe $^{37}$ & 11 & $\begin{array}{l}\text { Multiple lacunes, with/without } \\
\text { pre-stroke dementia }\end{array}$ & Clinical diagnosis & $>1$ month & 27 \\
\hline Miyao $^{38}$ & 215 & $\begin{array}{l}\text { First stroke, no pre-stroke } \\
\text { dementia }\end{array}$ & $\begin{array}{l}\text { DSM-III-R \& MMSE } \\
<20\end{array}$ & 2 years & 11.2 \\
\hline Samuelsson ${ }^{39}$ & 81 & First stroke & Clinical diagnosis & 3 years & 11 \\
\hline Desmond $^{40}$ & 170 & $\begin{array}{l}\text { With/without previous stroke } \\
\text { or pre-stroke dementia }\end{array}$ & DSM-III-R & 3 months & 25.9 \\
\hline Present study & 75 & $\begin{array}{l}\text { With/without previous stroke } \\
\text { or pre-stroke dementia }\end{array}$ & $C D R \geqslant 1$ & 3 months & 13.3 \\
\hline
\end{tabular}

also be present among our controls. Furthermore, other nonvascular factors may be affecting the cognition of patients with SSVD who have no cognitive symptoms. Hence, further study is needed to evaluate the relation between ischaemic lesions and cognitive impairment both in controls and in patients with SSVD who have no cognitive symptoms.

On the whole, patients with CDR of 0 or 0.5 had mild stroke severity and minimal functional impairment. The immediate relevance of detecting patients with mildly disabling cognitive symptoms or mild impairment in the performance of psychometric tests but without cognitive symptoms is uncertain. However, as cognitive impairment in small vessel disease has a propensity to progress, ${ }^{10}$ it is of paramount importance to study any potentially reversible risk factors that are associated with further cognitive deterioration among this group of patients.

We had excluded 17 patients with relevant small infarcts but who had unknown vascular aetiology or who had not participated in the cognitive assessment. As these patients were significantly older than the analysed subjects, we may have underestimated the frequency of cognitive impairment in our present study. Furthermore, patients with major depression were also among those who were excluded $(\mathrm{n}=3)$. It is currently believed that subcortical lesions, cognitive impairment, and depression are all inter-related. ${ }^{42}$ Hence, our estimation on the frequency of the varying severity of cognitive impairment was only conservative.

\section{Determinants for $C D R \geqslant 1$}

Pre-stroke cognitive decline and previous stroke were identified to predict severe cognitive impairment 3 months after SSVD. The cause for pre-stroke cognitive decline has not been investigated in the present study. Understanding this cause is important to the management of post-stroke cognitive impairment, as the disease process that has been affecting the patients pre-stroke is probably continuing to affect them post-stroke. Studies on pre-stroke cognitive decline among patients with stroke in general show that both ischaemic and degenerative pathology predict pre-stroke cognitive decline. ${ }^{43-45}$ Further study is needed to explore the aetiology and determinants of pre-stroke cognitive decline among patients with SSVD. It is interesting to find that previous stroke, rather than ischaemic lesions (WMC, small infarcts) predictes post-stroke severe cognitive impairment in SSVD. Other variables that have not been explored in the present study may be associated with previous stroke and with post-stroke cognitive impairment. A recent study suggests that atrophy of the cortex and hippocampus, which can only be partially explained by WMC or Alzheimer's pathology, was associated with dementia in patients with subcortical lacunes who were recruited randomly from a dementia clinic. ${ }^{46}$ It may be worthwhile to explore the relation between previous stroke, atrophy of the cortex or hippocampus, and post-stroke severe cognitive impairment in SSVD. We must emphasise that caution should be taken in interpreting the results from the regression analysis. As our sample size was small, the confidence intervals of the two variables identified as significant were wide (table 3 ). A larger study is needed to confirm the importance of prestroke cognitive decline and previous stroke in predicting post-stroke severe cognitive impairment in SSVD.

\section{Functional outcome}

Multivariate regression analysis demonstrated that performance in basic ADL as measured by BI was significantly associated with stroke severity (NIHSS) in our present cohort. This is consistent with other studies of SSVD showing that motor impairment significantly predicts physical dependence. ${ }^{15} 39$ Other variables that have been shown to predict physical dependence in SSVD are WMC, age, diabetes, previous stroke, and type of lacunar syndrome. ${ }^{15} 39$ However, the influence of cognitive impairment on the performance of complex ADL in SSVD has rarely been studied. We have demonstrated that stroke severity and performance in MDRS I/P contributes most to the variance in IADL. Age and pre-stroke IQCODE has a lesser contribution to its variance. As MDRS I/P evaluates executive dysfunction, our findings suggest that executive dysfunction predicts impairment in complex ADL among patients with SSVD. Tests that are biased towards evaluation of memory and language impairment, namely MMSE and ADAS-cog, were found not to be associated with performance of complex ADL in our subjects. A recent study also suggests that executive dysfunction among stroke patients in general is associated with reduced performance in both basic and complex ADL..$^{53}$ Our findings highlight the importance of cognitive impairment, and in particular, executive dysfunction, in affecting the functional outcome of patients with SSVD.

\section{Authors' affiliations \\ V C T Mok, A Wong, W W M Lam, Y H Fan, W K Tang, T Kwok, A C F Hui, K S Wong, The Chinese University of Hong Kong, Hong Kong, China}

Competing interests: none declared

\section{REFERENCES}

1 Erkinjuntti T, Inzitari D, Pantoni L, et al. Research criteria for subcortical vascular dementia in clinical trials. J Neural Transm 2000(Suppl);59:23-30.

2 Roman GC, Erkinjuntti T, Wallin A, et al. Subcortical ischemic vascular dementia. Lancet Neurol 2002;1:426-36.

3 Tatemichi TK, Desmond DW, Prohovnik I, et al. Confusion and memory loss from capsular genu infarction: a thalamcortical disconnection syndrome? Neurology 1992;42:1966-79.

4 Cummings JL. Frontal-subcortical circuits and human behavior. Arch Neurol 1993;50:873-80. 
5 Kramer JH, Reed BR, Mungas D, et al. Executive dysfunction in subcortical ischemic vascular disease. J Neurol Neurosurg Psychiatry 2002;72:217-20.

6 Naeser MA, Alexander MP, Helm-Estabrooks, et al. Aphasia with predominantly subcortical lesion sites. Arch Neurol 1982;39:2-14.

7 Giroud M, Lemesle M, Madinier G, et al. Unilateral lenticular infarcts: radiological and clinical syndromes, aetiology, and prognosis. J Neurol Neurosurg Psychiatry 1997;63:611-15.

8 Babikian V, Ropper AH. Binswanger's disease: a review. Stroke 1987; 18:2-12.

9 Meyer JS, Xu G, Thornby J, et al. Is mild cognitive impairment prodromal for vascular dementia like Alzheimer's disease? Stroke 2002;33:1981-5.

10 Aharon-Peretz J, Daskovski E, Mashiach T, et al. Natural history of dementia associated with lacunar infarctions. J Neurol Sci 2002;203-204:53-5.

11 Korczyn AD. Mixed dementia-the most common cause of dementia. Ann NY Acad Sci 2002;977:129-34.

12 Bamford J, Sandercock P, Jones L, et al. The natural history of lacunar infarction: The Oxfordshire Community Stroke Project. Stroke 1987; 18:545-51.

13 Sacco SE, Whisnant JP, Broderick JP, et al. Epidemiological characteristics of lacunar infarcts in a population. Stroke 1991;22:1236-41.

14 Rothrock JF, Lyden PD, Brody ML, et al. An analysis of ischemic stroke in an urban Southern California population. Arch Intern Med 1993; 153:619-24.

15 Clavier I, Hommel M, Besson G, et al. Long term prognosis of symptomatic lacunar infarcts-a hospital-based study. Stroke 1994;25:2005-9.

16 Wong KS, Gao S, Chan YL, Ringelstein EB, et al. Mechanisms of acute cerebral infarctions in patients with middle cerebral artery stenosis: $A$ diffusion-weighted imaging and microemboli monitoring study. Ann Neuro 2002:52:74-81.

17 Wong KS, Li H, Chan YL, et al. Use of transcranial Doppler ultrasound to predict outcome in patients with intracranial large-artery occlusive disease. Stroke 2000;31:2641-7

18 American Psychiatric Association. Diagnostic and statistical manual disorders, 4th ed. (DSM-IV). Washington, DC: American Psychiatric Association, 1994.

19 Wahlund LO, Barkhof F, Fazekas F, et al. on behalf of the European Task Force on Age Related White Matter Changes. A new rating scale for agerelated white matter changes applicable to MRI and CT. Stroke 2001;32:1318-22.

20 Huges CP, Berg L, Danziger L, et al. A new clinical scale for the staging of dementia. Br J Psychiatry 1982; 140:566-72.

21 Morris JC. The clinical dementia rating (CDR): current version and scoring rules. Neurology 1993;43:2412-14.

22 Chiu HFK, Kee HC, Chung WS, et al. Reliability and validity of the Cantonese version of Mini-mental state examination-a preliminary study. J Hong Kong Coll Psychiatry 1994:4(Suppl 2):25-8.

23 Chu LW, Chiu KC, Hui SL, et al. The reliability and validity of the Alzheimer's disease assessment scale cognitive subscale (ADAS-cog) among the elderly Chinese in Hong Kong. Ann Acad Med Singapore 2000;29:474-85.

24 Chan AS, Choi MK, Salmon DP. The effects of age, education, and gender on the Mattis dementia rating scale performance of elderly Chinese and American individuals. J Gerontol Psychol Sci 2001;56B:356-63.

25 Mungas D, Jagust WJ, Reed BR, et al. MRI predictors of cognition in subcortical ischemic vascular disease and Alzheimer's disease. Neurology 2001;57:2229-35.
26 Fuh JL, Teng EL, Lin KN, et al. The Informant Questionnaire on Cognitive Decline in the Elderly (IQCODE) as a screening tool for dementia for a predominantly illiterate Chinese population. Neurology 1995;45:92-6.

27 Lawton MP, Brody EM. Assessment of older people: self-maintaining and instrumental activities of daily living. Gerontologist 1969;9:179-86.

28 Bamford JM, Warlow CP. Evolution and testing of the lacunar hypothesis. Stroke 1988; 19:1074-82

29 Baumgartner RW, Sidler C, Mosso M, et al. Ischemic lacunar stroke in patients with and without potential mechanism other than small-artery disease. Stroke 2003:34:653-9.

30 Van Zandvoort MJE, Kappelle $\sqcup$, Algra A, et al. Decreased capacity for mental effort after single supratentorial lacunar infarct may affect performance in everyday life. J Neurol Neurosurg Psychiatry 1998;65:697-702.

31 Caplan LR. Caudate infarcts. In: Donnan G, Norrving B, Bamford J, Bogousslavsky J, eds. Subcortical stroke, 2nd edn. New York: Oxford University Press, 2002:209-23.

32 de Freitas GR, Bogousslavsky J. Thalamic infarcts. In: Donnan G, Norrving B, Bamford J, Bogousslavsky J, eds. Subcortical stroke, 2nd edn. New York: Oxford University Press, 2002:255-85.

33 Malm J, Kristensen B, Karlsson T, et al. Cognitive impairment in young adults with infratentorial infarcts. Neurology 1998;51:433-40.

34 Bowler JV. The concept of vascular cognitive impairment. J Neurol Sci 2002;203:11-15.

35 Mungas D, Reed BR, Jagust WJ, et al. Volumetric MRI predicts rate of cognitive decline related to $A D$ and cerebrovascular disease. Neurology 2002; 59:867-73

36 Tatemichi TK, Foulkes MA, Mohr JP, et al. Dementia in stroke survivors in the stroke data bank cohort, prevalence, incidence, risk factors, and computed tomographic findings. Stroke 1990;21:858-66.

37 Wolfe N, Linn R, Babikian VL, et al. Frontal systems impairment following multiple lacunar infarcts. Arch Neurol 1990;47:129-32.

38 Miyao S, Takano A, Teramoto J, et al. Leukoaraiosis in relation to prognosis for patients with lacunar infarction. Stroke 1992;23:1434-8.

39 Samuelsson $M$, Soderfeldt B, Olsson GB. Functional outcome in patients with lacunar infarction. Stroke 1996;27:842-6.

40 Desmond DW, Moroney JT, Paik MC, et al. Frequency and clinical determinants of dementia after ischemic stroke. Neurology 2000;54:1124-31.

41 Schmidt R, Fazekas F, Offenbacher $\mathrm{H}$, et al. Neuropsychologic correlates of MRI white matter hyperintensities: a study of 150 normal volunteers. Neurology 1993:43:2490-4.

42 Carota A, Staub F, Bogousslavsky J. Emotions, behaviours and mood changes in stroke. Curr Opin Neurol 2002; 15:57-69.

43 Henon H, Pasquier F, Durieu I, et al. Preexisting dementia in stroke patients. Baseline frequency, associated factors, and outcome. Stroke 1997:28:2429-36.

44 Pohjasvaara T, Mantyla R, Aronen HJ, et al. Clinical and radiological determinants of prestroke cognitive decline in a stroke cohort. $J$ Neurol Neurosurg Psychiatry 1999;67:742-8.

45 Barba R, Castro MD, del Mar Morin M, et al. Prestroke dementia. Cerebrovasc Dis 2001;11:216-24.

46 Fein G, Di Sclafani V, Tanabe J, et al. Hippocampal and cortical atrophy predict dementia in subcortical ischemic vascular disease. Neurology 2000;55: 1626-35

47 Pohjasvaara T, Leskela $M$, Vataja $R$, et al. Post-stroke depression, executive dysfunction and functional outcome. Eur J Neurol 2002;9:269-75. 